Article

\title{
Optimized Spectrometers Characterization Procedure for Near Ground Support of ESA FLEX Observations: Part 1 Spectral Calibration and Characterisation
}

\author{
Laura Mihai ${ }^{1, *(\mathbb{D})}$, Alasdair Mac Arthur ${ }^{2}$, Andreas Hueni ${ }^{3}$ (D) , Iain Robinson ${ }^{4}$ and Dan Sporea ${ }^{1}$ \\ 1 CETAL, Photonic Investigations Laboratory, National Institute for Laser, Plasma and Radiation Physics, \\ Măgurele 77125, Romania; dan.sporea@inflpr.ro \\ 2 School of Geosciences, University of Edinburgh, Edinburgh EH9 3FF, UK; alasdair.macarthur@ed.ac.uk \\ 3 Remote Sensing Laboratories, Department of Geography, University of Zurich, 8057 Zurich, Switzerland; \\ andreas.hueni@geo.uzh.ch \\ 4 Rutherford Appleton Laboratory Space Science and Technology Department, Harwell Campus, Didcot, \\ Oxfordshire OX11 0QX, UK; sdfkl239874@googlemail.com \\ * Correspondence: laura.mihai@inflpr.ro
}

Received: 31 December 2017; Accepted: 10 February 2018; Published: 13 February 2018

\begin{abstract}
The paper presents two procedures for the wavelength calibration, in the oxygen telluric absorption spectral bands $\left(\mathrm{O} 2-\mathrm{A}, \lambda_{\mathrm{c}}=687 \mathrm{~nm}\right.$ and $\left.\mathrm{O} 2-\mathrm{B}, \lambda_{\mathrm{c}}=760.6 \mathrm{~nm}\right)$, of field fixed-point spectrometers used for reflectance and Sun-induced fluorescence measurements. In the first case, $\mathrm{Ne}$ and Ar pen-type spectral lamps were employed, while the second approach is based on a double monochromator setup. The double monochromator system was characterized for the estimation of errors associated with different operating configurations. The proposed methods were applied to three Piccolo Doppio-type systems built around two QE Pros and one USB2 + H16355 Ocean Optics spectrometers. The wavelength calibration errors for all the calibrations performed on the three spectrometers are reported and potential methodological improvements discussed. The suggested calibration methods were validated, as the wavelength corrections obtained by both techniques for the QE Pro designed for fluorescence investigations were similar. However, it is recommended that a neon emission line source, as well as an argon or mercury-argon source be used to have a reference wavelength closer to the O2-B feature. The wavelength calibration can then be optimised as close to the O2-B and O2-A features as possible. The monochromator approach could also be used, but that instrument would need to be fully characterized prior to use, and although it may offer a more accurate calibration, as it could be tuned to emit light at the same wavelengths as the absorption features, it would be more time consuming as it is a scanning approach.
\end{abstract}

Keywords: ESA Fluorescence Explorer (FLEX) mission; field fixed-point spectrometers; oxygen telluric absorption lines; Sun-induced fluorescence; wavelength calibration

\section{Introduction}

Fluorescence, the radiant flux emitted by chlorophyll molecules after excitation by photons, is the most directly-measurable reporter of photosynthetic efficiency and hence a key indicator of the health and carbon fixation of photosynthesizing organisms [1,2]. Measurements of fluorescence, at both the leaf and canopy level, can therefore greatly advance the understanding of the photosynthesis dynamics, gross primary productivity and ecosystem change over time [3,4]. A synoptic view of Earth vegetation health will become available when the ESA Fluorescence Explorer (FLEX) mission, scheduled for launch in 2022, becomes operational. This mission will fly in tandem with Sentinel 2 and 3 and enable both the Sun-induced fluorescence (SIF) and Earth surface radiance to be measured 
simultaneously [5-7]. Reliable short-term and long-term system characterization, calibration and validation of the spectrometer system used for ground and near ground calibration and validation science activities [8-11] need to be considered to ensure accurate and replicable results.

SIF investigations are challenging due to the extremely low levels of light produced by chlorophyll emissions, as these are overlapped by vegetation solar flux reflectance signals. One of the main strategies used to separate the effects of vegetation reflected and emitted fluxes is by exploiting the changes across the oxygen telluric absorption lines: oxygen-A (O2-A), having the centre wavelength at $687 \mathrm{~nm}$ and oxygen-B (O2-B) having the centre wavelength at $760.6 \mathrm{~nm}[12,13]$. These methods require the use of high spectral resolutions spectrometers (full-width half-maximum (FWHM) of less than $0.3 \mathrm{~nm}$ ) $[13,14]$. The need for high-resolution spectrometers for measuring fluorescence within these telluric bands and the effects of inaccuracies and uncertainties has been highlighted by [15-17], and the need for well-calibrated and characterised systems is emphasised in the ESA FLEX report for mission selection [3]. The impact of different spectrometer FWHMs on the shape of the O2-A absorption feature and shift in the apparent minima are shown in Figure 1, as an example. Consequently, field spectrometers require very careful spectral calibration (wavelength position and, hence, sampling interval) and characterization (FWHM) to demonstrate that these requirements are met. In addition, attention needs to be paid to the temperature of the spectrometer. Differences between temperatures during spectrometer calibration and field use may also introduce additional uncertainties in spectral calibration [18]. The work reported here was carried out at in a laboratory at $20{ }^{\circ} \mathrm{C}$ and with each QEPro spectrometer detector cooled to $-10{ }^{\circ} \mathrm{C}$. In general, field fixed-point spectrometers, covering the $400 \mathrm{~nm}-1100 \mathrm{~nm}$ or $400 \mathrm{~nm}-2500 \mathrm{~nm}$ spectral ranges, are used for ground-based calibration and validation of space-based observations [19-23]. However, although a few independent studies have been published on high-resolution field spectrometers' performance characterization and calibration [24,25], these studies are not directly comparable as the methodologies and instruments varied.

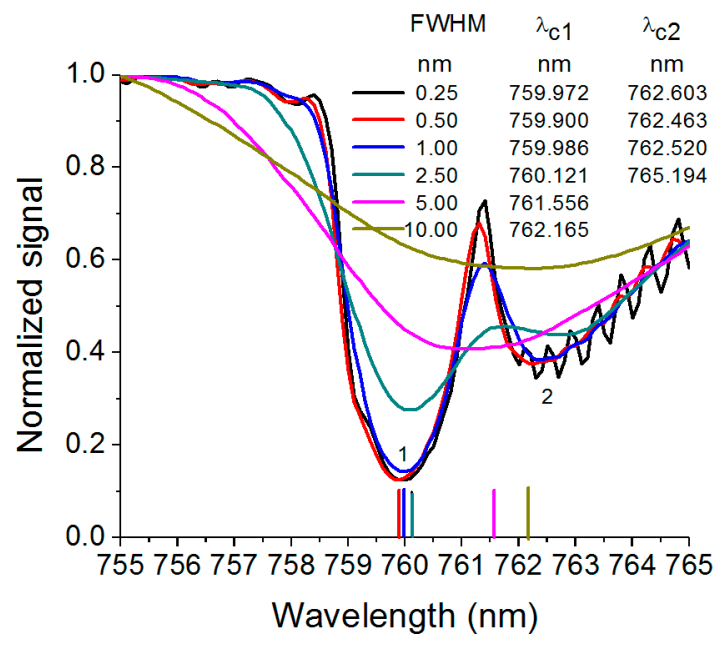

Figure 1. The sunlight signal intensity measured in the spectral range 755-765 nm with the spectral calibration system, an OL-750D double monochromator from Gooch \& Housego (Orlando, FL, USA), using different slit widths. The vertical lines represent the positions of the centre wavelengths associated with specific configurations of the double monochromator. The same colour coding was used for wavelength positions corresponding to the FWHM values. Original radiometrically-calibrated data provided by Chris MacLellan, NERC Field Spectroscopy Facility, University of Edinburgh, U.K.

As of yet, no detailed and universally-accepted protocols have been developed for the calibration of field spectrometers. Spectrometer manufacturers do not normally release details of their calibration procedures, claiming commercial confidentiality, or require non-disclosure agreements (NDA) to be signed, if they do provide any information. Their work cannot therefore be more generally replicated. 
Consequently, each research group has to develop its own calibration procedures. Calibration protocols have also not yet been developed for the even higher spectral resolution field spectrometers, which will be required for calibration, validation and science activities to support the FLEX fluorescence imager at sufficient spectral sampling intervals and band widths to measure solar flux in the telluric O2-A and O2-B bands. Therefore, primary calibration sources traceable to agreed common standards and laboratory calibration protocols are required for reliable and replicable measurements and to enable measurements from one near-ground system to be compared directly with measurements from other systems. Detailed protocols for radiometric, geometric and spectral calibration are required to ensure that this is the case. The spectral calibration refers to spectrometer detector spectral response with reference to a known wavelength emission standard [25]. Ocean Optics (Dunedin, FL, USA), a company providing high-resolution optical benches, which can be incorporated into spectrometer systems for field use, recommend calibration against a mercury argon ( $\mathrm{Hg}-\mathrm{Ar}$ ) emission source and a third order polynomial fit to generate the wavelength calibration scale [26]. Nevertheless, a consistent and replicable approach needs to be agreed upon, given the errors that can be introduced in fluorescence estimation [27].

Different spectral standards (emission/absorption sources, monochromator-based systems or tuneable laser sources) can be used as wavelength standards, depending on the spectral measurement resolution (FWHM and the sampling interval) required [16-29]. The most frequently-used spectral source for this purpose is the pencil style calibration lamps (L.O.T-Oriel, U.K., for example), having very narrow emission lines, with a line width $<0.001 \mathrm{~nm}$ [30-32]. The disadvantages when using these types of emission lamps is the limited emission line distribution across the spectral range that corresponds to the gas chemistry and physics fundamentals. These lines are not necessarily evenly distributed across the full range of the spectrometers being calibrated, and their intensity at some wavelengths may be too low to be of practical use [29]. In addition, the emission lines do not normally match the wavelengths corresponding to O2-A and O2-B absorption lines. Another option is to use a tuneable monochromator system. These systems, which can be programmed to emit light at specific wavelengths in minimum steps of $0.05 \mathrm{~nm}$, depending on the configurations, can be tuned to provide FWHMs between $<0.25 \mathrm{~nm}$ and up to $10 \mathrm{~nm}$. When operated in conjunction with a high intensity light source, they can provide a signal intensive enough to be detected by field spectrometers being used for fluorescence studies. One disadvantage of such systems is that their operation can be time consuming when a large spectral range needs to be considered for spectral calibration [29]. Another type of source that can be used for spectral calibration is a tuneable laser that can provide multiple intense emission [8,29]; however, this approach is out of the scope of this study; as a system was not available for this work. However, the use of a tuneable laser for spectral calibration is planned for a future research project. This paper will discuss two different laboratory spectral calibration techniques (using narrow band pen-line emission sources and a double monochromator-based standard) applied to field spectrometers with different spectral ranges, sampling intervals and FWHMs. The spectrometers' calibration accuracy necessarily depends on the calibration equipment performances, and since the spectral response can vary with different configurations, a proper characterization of this equipment is critical [8].

The focus of our study was to compare wavelength calibration approaches and develop recommendations for the spectral characterization of: high and low-resolution spectrometers prior to developing appropriate procedures and protocols. Therefore, the evaluation of a Piccolo spectrometer system spectral response was selected. This instrument is a dual-field-of-view system able to incorporate multiple optical benches (individual spectrometers covering different spectral ranges) with a cosine-corrected fore optic to capture downwelling irradiance and the upwelling channel configured with a view angle-limited fore optic [33]. Light is transmitted from the fore optics to each individual spectrometer through a bifurcated fibre optic assembly (Figure 2). This assembly has two fibre optic cables, in each of its input legs (one leg for upwelling light and the other for downwelling light), and these cross over at the central block so that one upwelling and one downwelling fibre go to each 
spectrometer. As there is an electro-mechanical shutter in each fore optic, one can be closed to exclude light entering the system while the other is open. Hence, by switching between the two shutters, downwelling and upwelling light can be recorded sequentially. The upwelling and the downwelling channel fibres are aligned along each spectrometer optical slit as shown in Figure 2. Consequentially, as the input geometry is different for each of these channels, each needs to have its wavelength calibrated. The spectrometers available for use with this system and investigated here are: (i) QE Pro spectrometer Serial Number QEP00114 (for fluorescence) with a $1044 \times 68$ 2D pixel array measuring across the spectral range $640 \mathrm{~nm}-800 \mathrm{~nm}$, providing a 0.15-nm sampling interval, and with a 10- $\mu \mathrm{m}$ slit, providing a 0.31-nm FHWM; (ii) QE Pro spectrometer Serial Number QEP0981 (for reflectance) with a $1044 \times 68$ $2 \mathrm{D}$ pixel array measuring across the spectral range $400-950 \mathrm{~nm}$ and with a $25-\mu \mathrm{m}$ slit providing around 2 nm FWHM; and (iii) USB2 + H16355 spectrometer (for reflectance) with a 2048 1D pixel array with a usable spectral range of $400 \mathrm{~nm}-950 \mathrm{~nm}$, a 0.4 sampling interval and with a $25-\mu \mathrm{m}$ slit providing around $1.5 \mathrm{~nm}$ FWHM. The QEPro 2D array pixel columns (68 pixels) are summed to provide an increased (200,000 usable counts) dynamic range. These spectrometers are provided by Ocean Optics (Dunedin, FL, USA) with a starting wavelength and a third order polynomial used to define the wavelength scale. The polynomial coefficients are generated from measurements of a $\mathrm{Hg}$-Ag emission line lamp with light being transferred to the spectrometer by a single core fibre optic assembly. Due to a different fibre optic assembly geometry (a bifurcated fibre optic) being used to transfer light to the spectrometer when incorporated into the Piccolo system, a wavelength recalibration is required. The manufacturers of the Piccolo also use a Hg-Ar lamp. A wavelength offset for each channel of the bifurcated fibre is determined and applied to all pixels in the wavelength scale for that channel. Reports of this correction and an assessment of accuracies are then provided by the suppliers of the Piccolo system (Figures 3 and 4 are examples) and demonstrate that the wavelength calibration of each channel of this system is the same; hence, there are no wavelength dependencies imposed by the downwelling channel cosine-corrected diffuser.

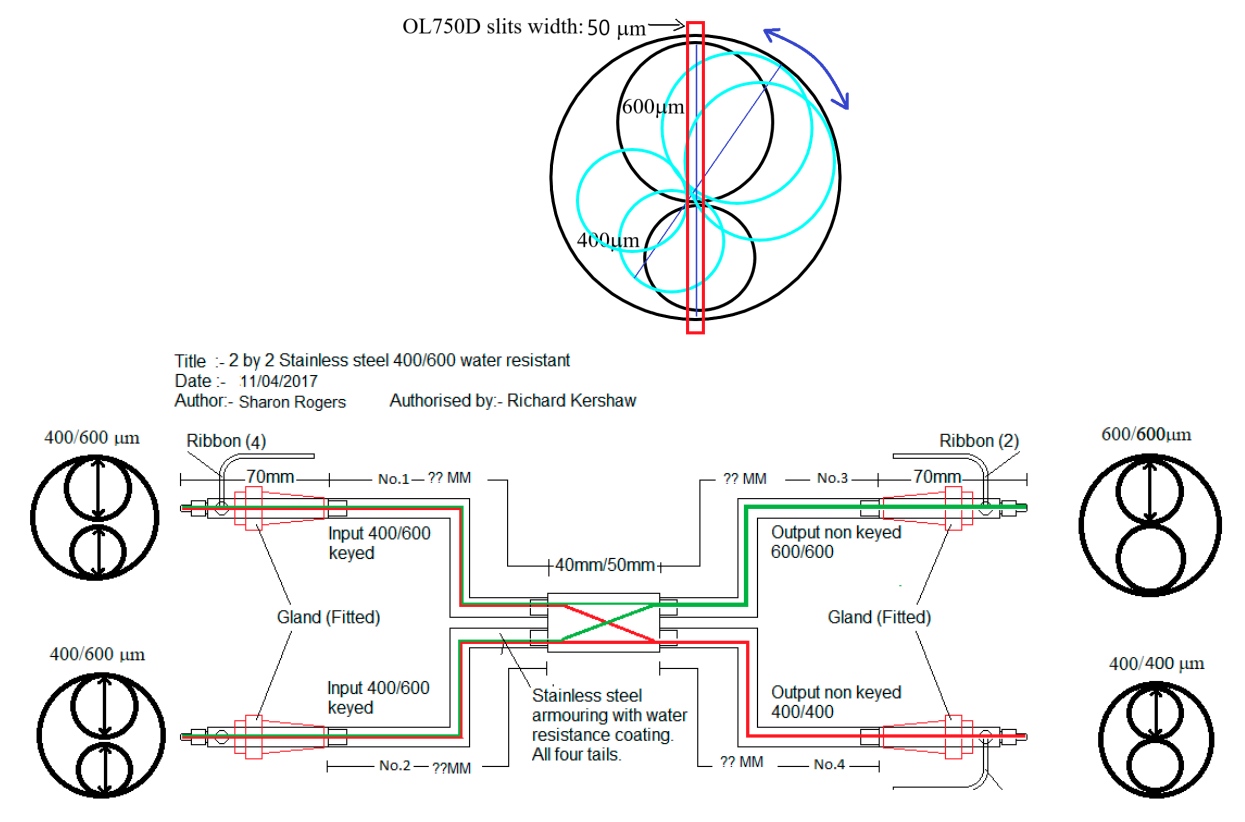

Figure 2. Schematic of the Piccolo system double bifurcated fore optic cable assembly showing the fibre core configuration. Misalignment of the fibre core with the spectrometer slit could lead to signal attenuation, and input geometry errors lead to geometry errors. 


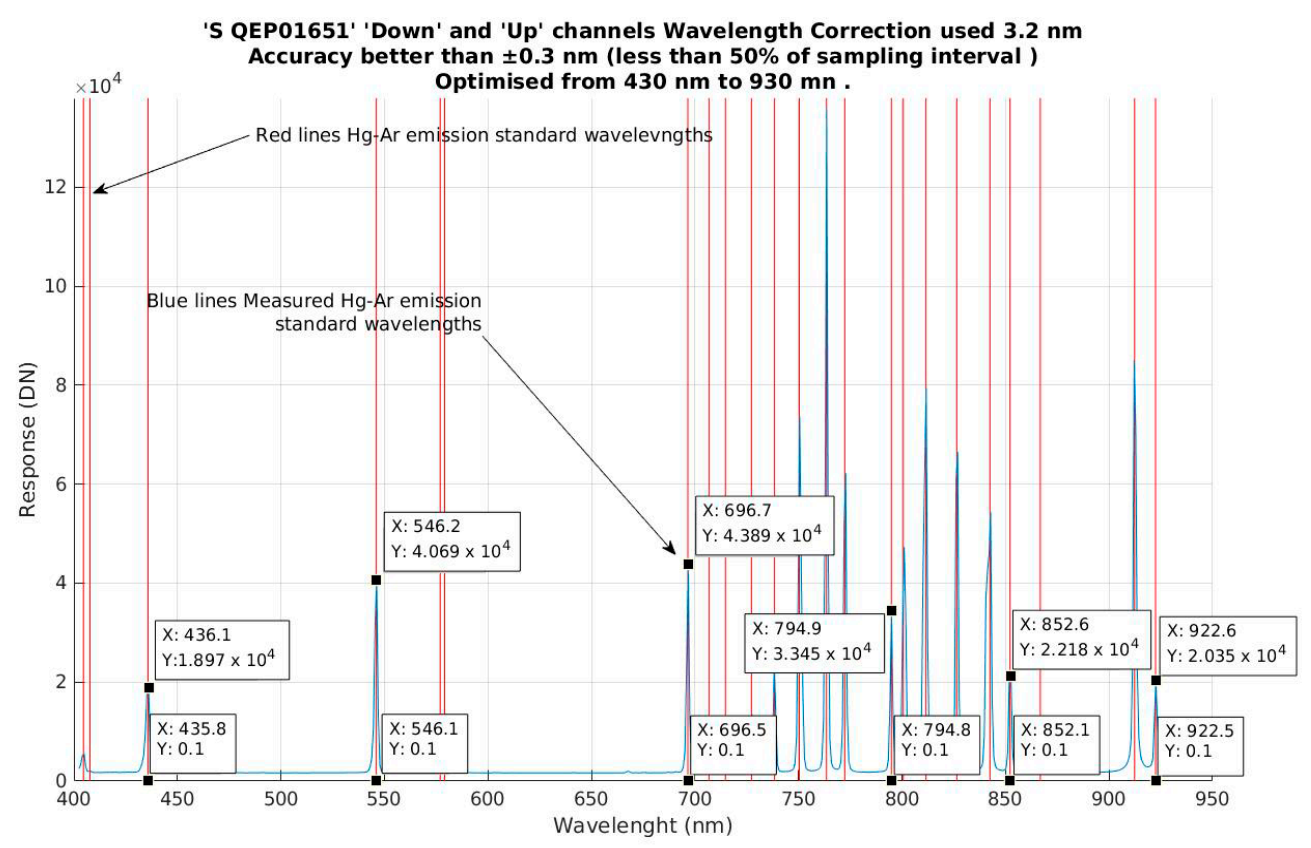

Figure 3. Report on Piccolo system calibration for QEPro 01651 as an example provided by Piccolo system suppliers.

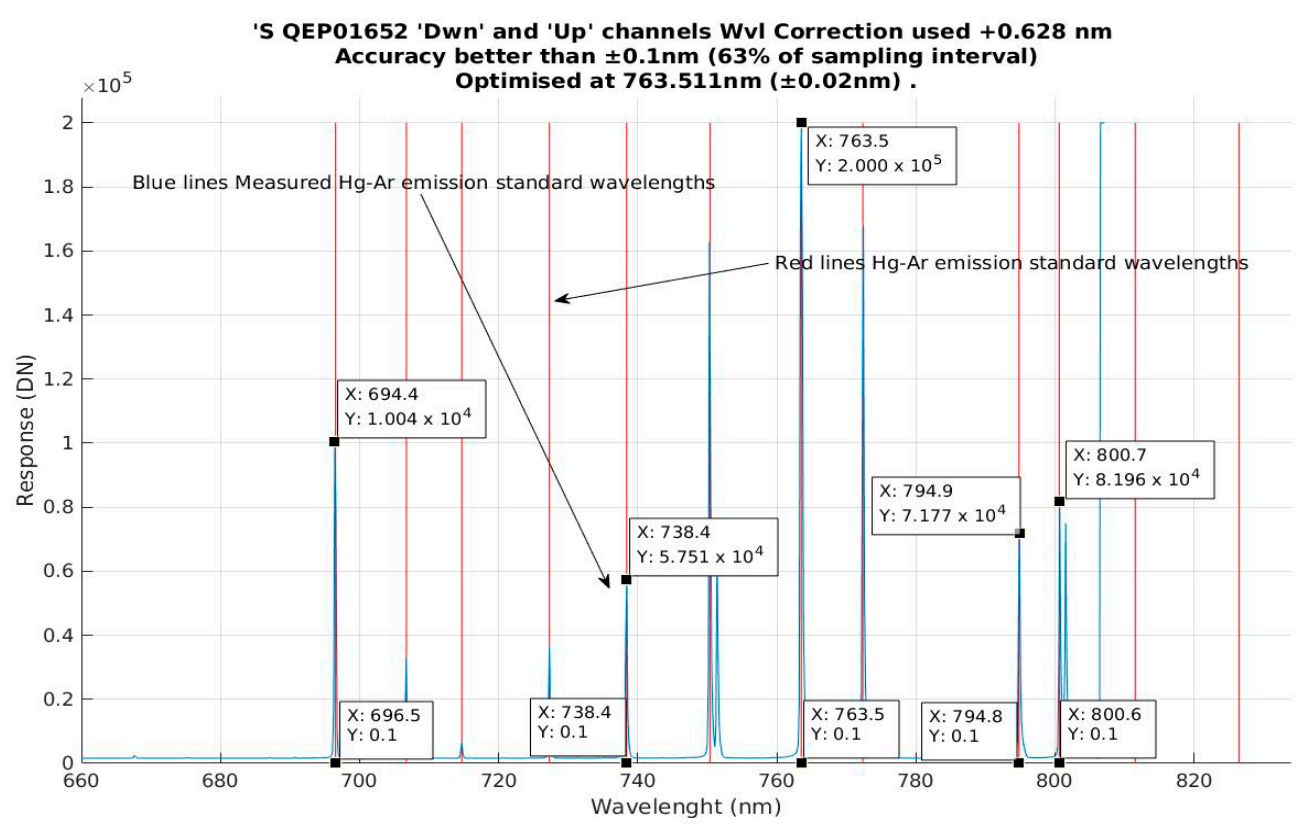

Figure 4. Report on Piccolo system calibration for QEPro 01652 as an example provided by Piccolo system suppliers.

However, it should be noted that neither Hg-Ar lamp nor argon (Ar) lamps available for this study have usable emission lines between $650 \mathrm{~nm}$ and $696 \mathrm{~nm}$. Therefore, the accuracy of the wavelength calibration at the $687 \mathrm{~nm}$ O2-B absorption feature cannot be verified by this method.

This paper investigates alternative methods of wavelength calibration. It presents the results obtained from testing the double monochromator performances in multiple configurations, as well as the measurements of a neon ( $\mathrm{Ne}$ ) pen-type emission source, compared to an Ar source. The advantages and disadvantages of these two calibration methods are then discussed and conclusions presented. 


\section{Methods}

The aim of our study was to identify the best practice related to wavelength calibration of high-resolution (narrow FWHM and high sampling interval) spectrometer systems across the telluric $\mathrm{O} 2-\mathrm{A}$ and O2-B bands using two different methods. As the wavelength calibration for each channel has been demonstrated to be the same (Figures 3 and 4) and as this work concerns methods rather than an absolute calibration, only one channel was investigated for each of the spectrometers. For the first approach, an Ar lamp was used, and the wavelength scale offset was determined in the same manner used by the Piccolo suppliers. For the first approach, each spectrometer (Serial Numbers QEP00114, QEP0981, USB2 + H16355) were incorporated into the Piccolo system and calibrated using an Ar lamp. Then, a Ne lamp was employed to assess the accuracy of this calibration at wavelengths closer to the O2-B absorption feature. The Ar lamp has more intense emission lines around $763 \mathrm{~nm}$, and the Ne lamp has intense emission lines below $700 \mathrm{~nm}$. These light sources are characterized by very narrow (line width below $0.001 \mathrm{~nm}$ ), intense and stable lines (important when calibrating high spectral resolution systems) produced by excitation of metal and gas vapours, having traceability to NIST standards [29,31]. The light from the gas lamps illuminated uniformly (through a 6" integrating sphere) one of the bifurcated optical fibre channels. First, the integrating sphere illuminated the fluorescence spectrometer (QEP0114) through the cosine-corrected fore optic, keeping the other fore optics covered with a dark coating. After that, reflectances spectrometers (QEP0981, USB2 + H16355) were illuminated using the view angle-limited fore optic, and the cosine diffuser was covered. Each optical fibre system input was aligned to the integrating sphere output using an optical fibre adapter (manufactured by an engineering company for this work) so that the light was normal to the optical fibre input. Ninety spectra were collected for each spectrometer test, with the integration time depending on the characterized system: $200 \mathrm{~ms}$ for QEP0114, 3 ms for USB2 + H16355 and $10 \mathrm{~ms}$ for QEP0981. All data were dark current corrected. The setup used for this method is represented in Figure 5.

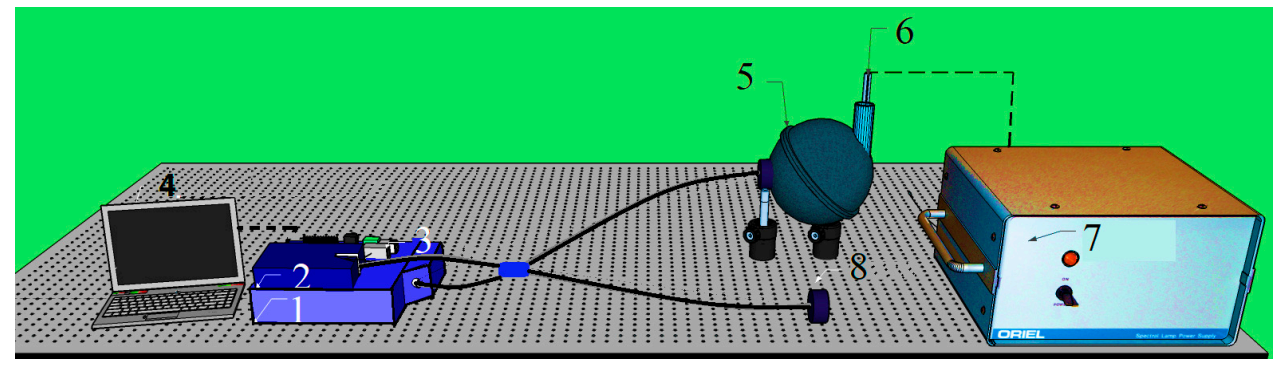

Figure 5. Piccolo Doppio characterization using as the light source multiple pencil-type reference lamps, with: (1) spectrometer QEPro; (2) spectrometer USB2000+ both from Ocean Optics; (3) Raspberry Pi system; (4) PC for spectrometers remote control; (5) NERC 6" integrating sphere optimized for pen-ray lamp entrance; (6) pen-ray emission lamp (Ne/Ar); (7) pen-ray lamps' power supply unit; (8) the covered optical fibre input for down-welling measurements; the dashed lines represent the power cables, and the continuous lines represent the optical fibres for signal collection.

The second method used as a wavelength standard is a high-efficiency grating double monochromator OL750D system from Gooch \& Housego (Orlando, FL, USA). This system can be used as an emission source for wavelength calibration (Figure 6), or it can be used as a detection source to measure light, when combined with a detector. As the standard, this laboratory instrument can provide monochromatic light from 200 up to $800 \mathrm{~nm}$ with a nominal mechanical resolution of $0.05 \mathrm{~nm}$ and tuneable full-width half-maximum below $0.25 \mathrm{~nm}$ (with the standard configuration: slits of $0.125 \mathrm{~mm}$, diffraction gratings 1800 grooves $/ \mathrm{mm}, 0.5-\mu \mathrm{m}$ blaze, FWHM of $0.15 \mathrm{~nm}$, tungsten lamp). However, the OL 750D used in this study had been modified by the Field Spectroscopy Facility at the University of Edinburgh in collaboration with Gooch \& Housego and configured with a high intensity light source (EX-99X LDSL from Energetiq, Woburn, MA, USA) and 0.05 mm, $0.5 \mathrm{~mm}$ and 
$0.05 \mathrm{~mm}$ slit widths at the monochromator entrance, middle and exits, respectively. The $0.05 \mathrm{~mm}$ slit size was chosen for the entrance and exit to enable the spectrometers spectral response to be characterized at a FWHM of less than $0.2 \mathrm{~nm}$. When mounting the optical fibre with proper optics at the monochromator exit, an accurate alignment has to be performed to reduce the errors due to setup geometry. The bifurcated optical fibre used to transmit the light to the spectrometer has two cores of different diameters (400 nm and $600 \mathrm{~nm}$ ). These cores therefore need to be aligned with the slit (Figure 2). For this work, after optimizing the optical fibre position to obtain the maximum signal at the spectrometer sensor, a precision engineered adapter with key to maintain alignment was used. The wavelength corrections for each spectrometer using this setup were determined as the difference between the wavelengths set at the monochromator controller and the spectrometers' readings. Therefore, the three spectrometers were spectrally characterized for all wavelength values within the $686 \mathrm{~nm}-688 \mathrm{~nm}$ and $759 \mathrm{~nm}-769 \mathrm{~nm}$ spectral ranges, in steps of $0.05 \mathrm{~nm}$. Similar to the calibration procedure using the pen-lamps, the light was guided to the fluorescence spectrometer through the cosine-corrected fore optic, keeping the other fore optics covered with a dark coating, and to the reflectance spectrometers through the view angle-limited fore optic, with the cosine diffuser covered (Figure 6).

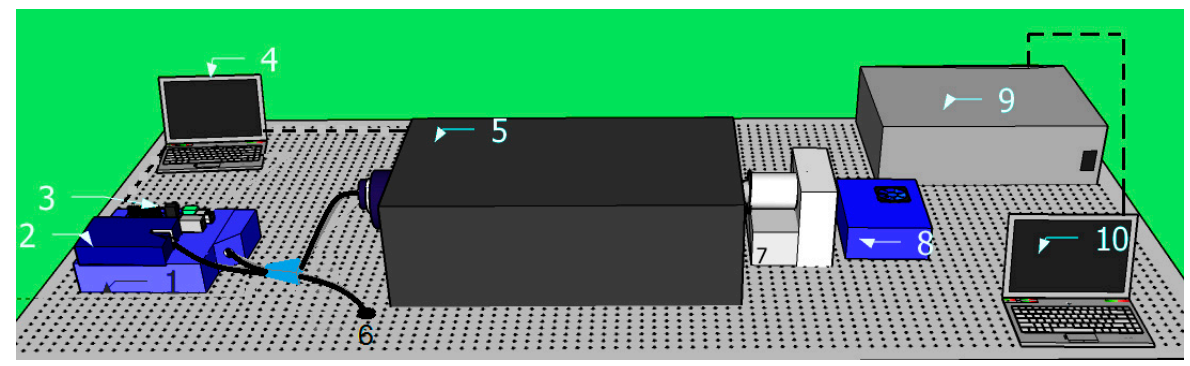

Figure 6. Piccolo Doppio characterization using as the light source a narrow band spectral calibration source, with: (1) spectrometer QEPro; (2) spectrometer USB2000+ both from Ocean Optics; (3) Raspberry Pi system; (4) PC for spectrometers remote control, (5) double monochromator OL750D from Gooch \& Housego; (6) the double bifurcated optical fibre system, with FOV-limited fore optics to one input and cosine corrected to the other input; (7) EX-99X LDSL high intensity light source; 8) the LDSL power supply controller unit; (9) OL750D controller; (10) PC for OL750D remote control.

In order to improve the accuracy and reliability of the results, some preliminary tests to check the double monochromator system performances using different configurations were carried out (Table 1 , Figure 7).

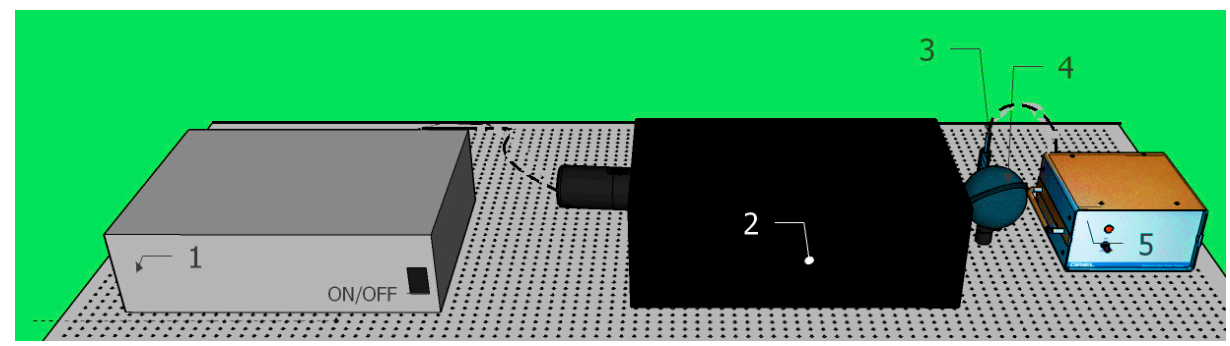

Figure 7. Narrow band spectral calibration source (OL750D double monochromator) characterization setup using multiple pencil-type reference lamps: (1) OL750D controller; (2) double monochromator OL750D from Gooch \& Housego; (3) pen-ray emission lamp (Ne/Ar); (4) NERC 6" integrating sphere optimized for pen-ray lamp entrance to improve the signal at the monochromator entrance; (5) pen-ray lamps' power supply unit. 
Table 1. Configurations selected for OL750D characterization.

\begin{tabular}{cccc}
\hline $\begin{array}{c}\text { Entrance, Middle, Exit Slits }+ \\
\mathbf{5 ~ m m} \text { Aperture at Exit }(\mathbf{m m})\end{array}$ & Reference Light Source & Spectral Range $(\mathbf{n m})$ & Sampling Interval (nm) \\
\hline $0.05,0.5,0.05$ & Neon & $686-696$ & 0.05 \\
$0.05,0.5,0.05$ & Argon & $758-768$ & 0.05 \\
$0.05,0.5,0.05$ & Argon & $768-778$ & 0.05 \\
$1.25,5.0,1.25$ & Neon & $686-696$ & 0.05 \\
$1.25,5.0,1.25$ & Argon & $758-768$ & 0.05 \\
$1.25,5.0,1.25$ & Argon & $768-778$ & 0.05 \\
$2.5,5.0,2.5$ & Neon & $686-696$ & 0.05 \\
$2.5,5.0,2.5$ & Argon & $758-768$ & 0.05 \\
$2.5,5.0,2.5$ & Argon & $768-778$ & 0.05 \\
$5.0,5.0,5.0$ & Neon & $686-696$ & 0.05 \\
$5.0,5.0,5.0$ & Argon & $758-768$ & 0.05 \\
$5.0,5.0,5.0$ & Argon & $768-778$ & 0.05 \\
\hline
\end{tabular}

Because the OL750D is a configurable monochromator, its wavelength calibration, sampling step and FWHM also had to be characterized as part of this work. As the emission lines from the $\mathrm{Ne}$ and Ar lamps are well defined, these were used as sources to characterize the monochromator. The spectral response characterization was performed in all cases (spectrometers and monochromator characterization), applying to the measured spectral response a Gaussian fit (Figure 8) defined in Equation (1):

$$
y=y_{0}+\left(\frac{A}{w * \sqrt{\frac{\pi}{2}}}\right) * e^{-2 * \frac{x-x_{c}}{w}}
$$

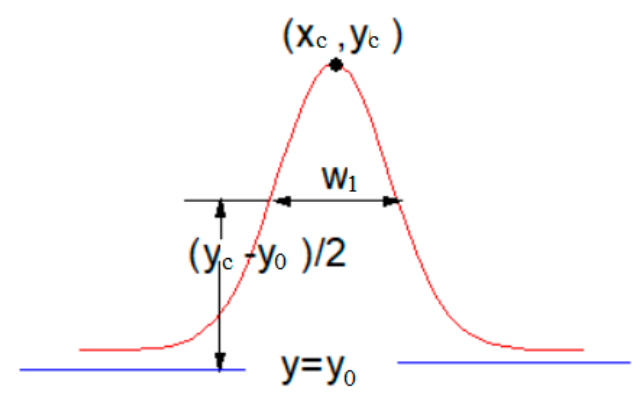

Figure 8. The sample curve corresponding to the Gaussian fit in Origin Lab.

Obtaining $x_{c}$, the central wavelength, and the FWHM $=\operatorname{sqrt}(2 * \ln (2)) * w$, where $w=w_{1} / \operatorname{sqrt}(\ln (4)$.

The wavelength error was determined as the difference between the wavelength values corresponding to the Ne and Ar emission lines specified in the NIST database $[34,35]$ and the centre wavelength obtained from the Gaussian fit applied to the measured spectral response using the field spectrometers. All wavelength correction values were obtained using the cubic B-spline interpolation function. This function splits data into multiple segments and fits each segment with Bezier splines [36]. The results are presented in the section bellow.

\section{Results}

\subsection{Field Spectrometers Spectral Characterization with Reference Emission Light Source}

The wavelength calibration and FWHM characterization of spectrometers QEP00114, QEP00981 and USB2H16355, using as spectral standard the Ar and Ne reference light sources, are presented hereafter. The centre wavelength and the FWHM for each peak was determined from the Gaussian fit, described above. The highest spectral resolution was obtained for the QEP00114, used for fluorescence 
measurements, with FWHM values between 0.3 and $0.43 \mathrm{~nm}$ depending on wavelength position, the other two spectrometers spectral resolutions being higher than $1.5 \mathrm{~nm}$ (Table 2). It was also noted that the wavelength sampling interval for each spectrometer decreased over the spectral range from $0.166 \mathrm{~nm}$ down to 0.132 for QEP00114, from $0.799 \mathrm{~nm}$ down to 0.684 for QEP00981 and from $0.374 \mathrm{~nm}$ down to $0.283 \mathrm{~nm}$ for USB2H16355.

The centre wavelength obtained from the Gaussian fit corresponding to the Ar 763.511-nm emission line was used to calculate the correction factor to be applied for the Piccolo bifurcated fibre optics to all wavelength values. The measured wavelength values for the device under test (DUT, in this case, the field spectrometer) represent the mean values of 90 acquired spectra, with a maximum spectral response Type A relative uncertainty of $0.009 \%$ along the measured spectral range. Therefore, correction factors of $+0.981 \mathrm{~nm},+2.055 \mathrm{~nm}$ and $-0.744 \mathrm{~nm}$ were applied to the QEP00114, QEP00981 and USB2H1635 spectrometers, respectively. The corrected spectra are presented in Figures 9, 10 and 11a,b and the associated errors, presented in Figures 9, 10 and 11c,d and Table 2. These errors were calculated as the difference between the reference Ar lamp standard wavelengths, $\lambda_{\text {standard }}$ (from NIST database) and $\lambda_{o}$, the measured wavelength optimized with the correction factor corresponding to the Ar lamp 763.511-nm emission line. Different error values were obtained across the spectral ranges, the highest differences being obtained for the QEP00981, and the lower for the fluorescence spectrometer QEP00114, when compared to the Ne lamp emission lines. The red lines in Figures 9-11 are the emission lines specific for the measured reference source.

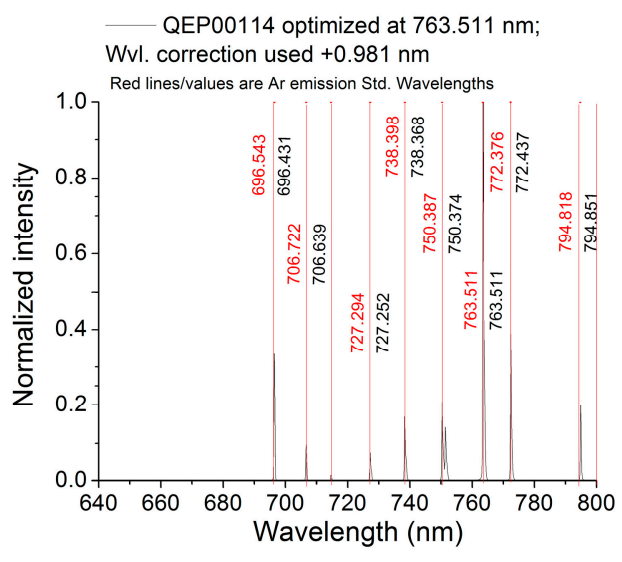

(a)

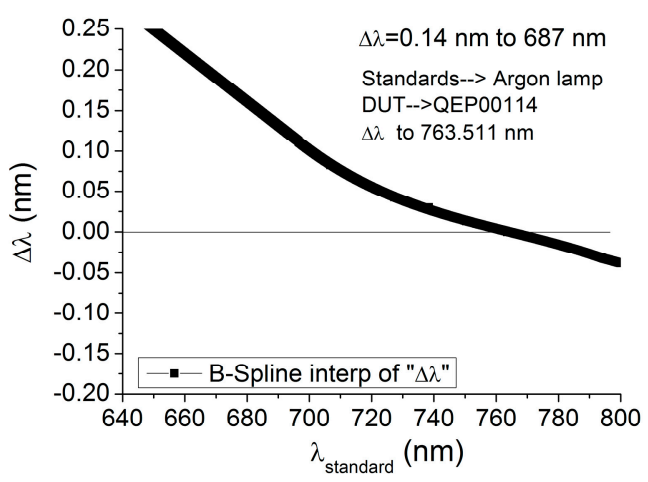

(c)

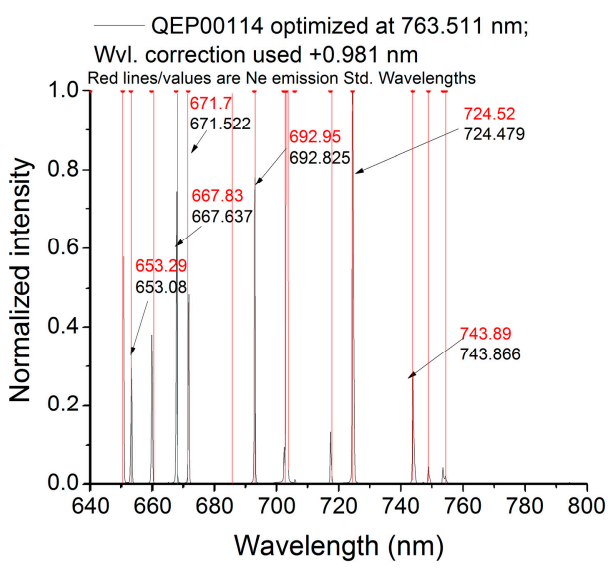

(b)

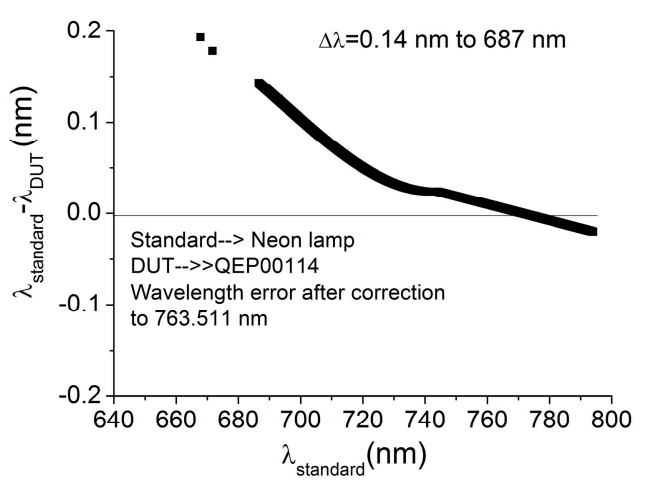

(d)

Figure 9. The wavelength corrections for (a) Ar and (b) Ne lamps' emission lines measured with the QEP00114 ( $\lambda_{\text {DUT }}$; DUT, device under test) after optimization at $763.511 \mathrm{~nm}$ and the corresponding errors $(\mathbf{c}, \mathbf{d})$ related to the standard values $\left(\lambda_{\text {standard }}\right)$. The red lines represent the standard emission lines for Ar and Ne lamps. 


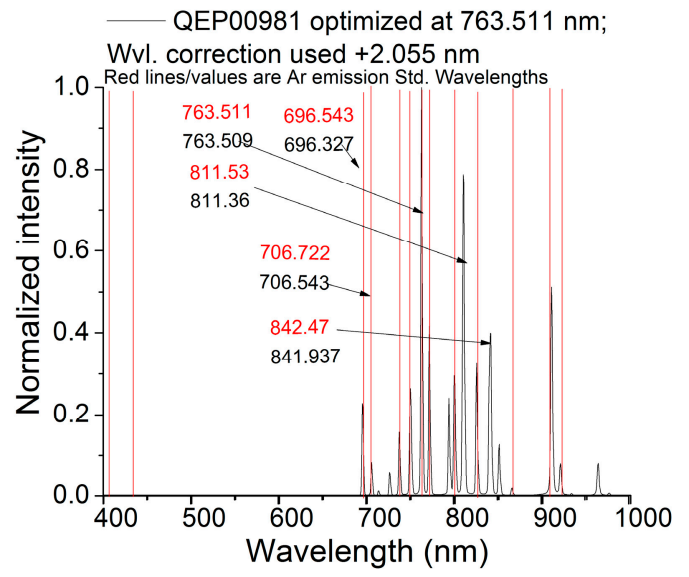

(a)

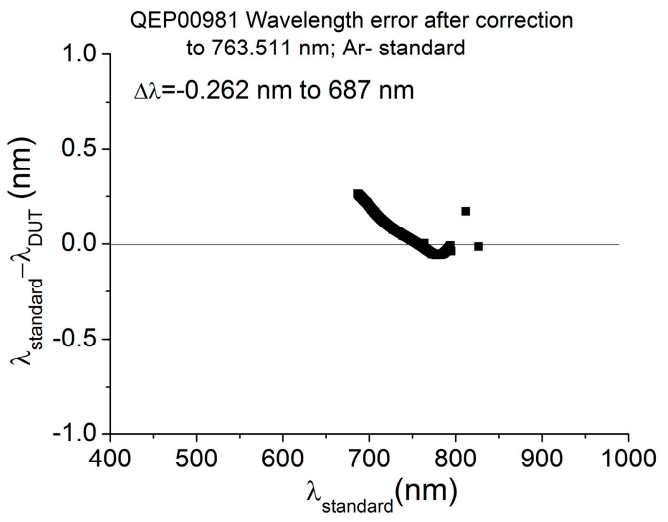

(c)

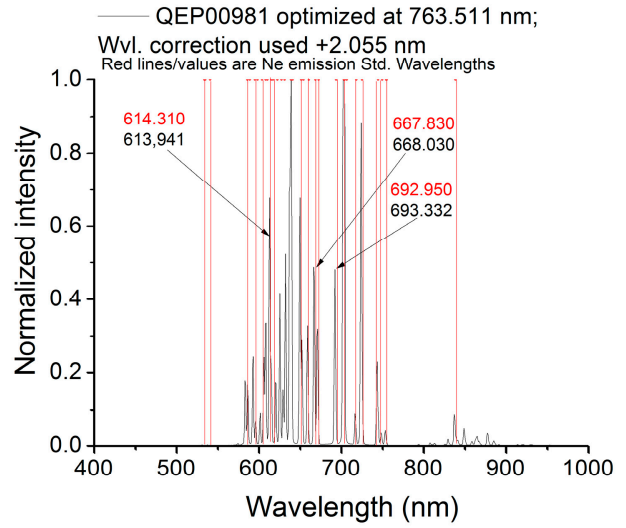

(b)

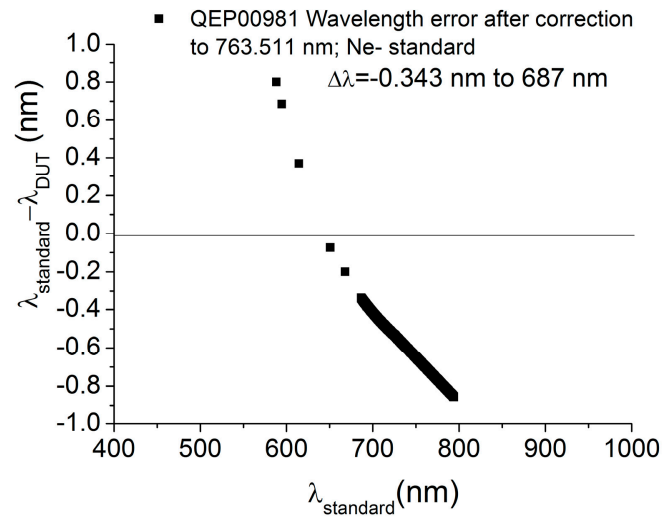

(d)

Figure 10. The wavelength corrections for (a) Ar and (b) Ne lamps' emission lines measured with the QEP00981 ( $\lambda_{\text {DUT }}$; DUT, device under test) after optimization at $763.511 \mathrm{~nm}$ and the corresponding errors $(\mathbf{c}, \mathbf{d})$ related to the standard values $\left(\lambda_{\text {standard }}\right)$. The red lines represent the standard emission lines for Ar and Ne lamps.

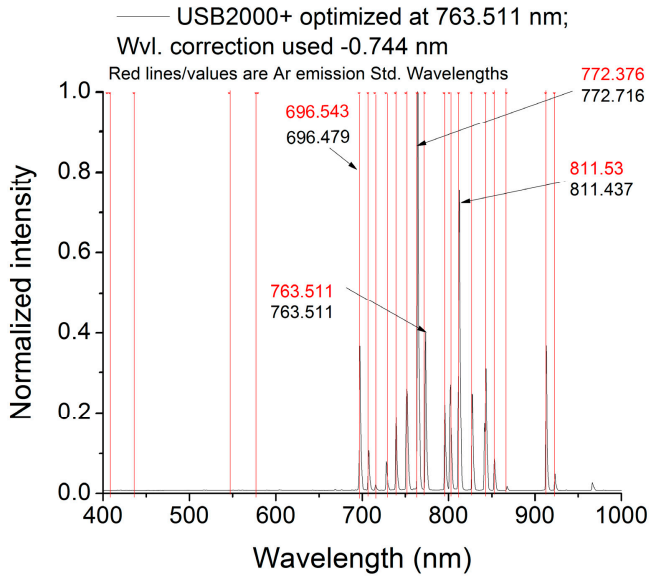

(a)

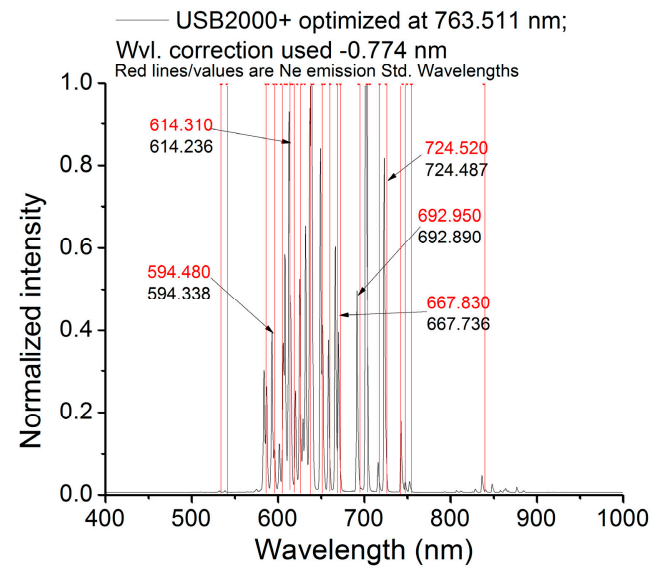

(b)

Figure 11. Cont. 


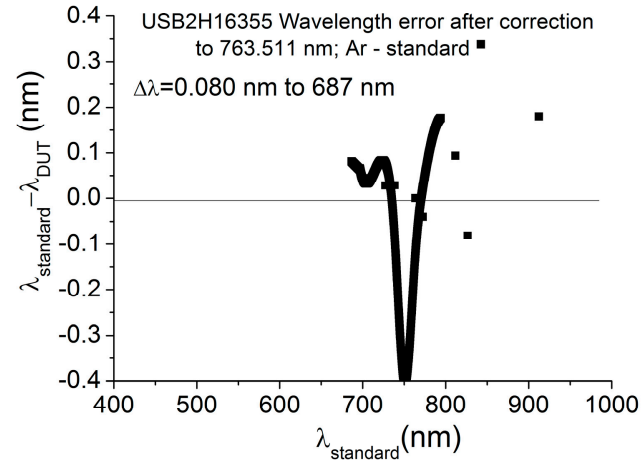

(c)

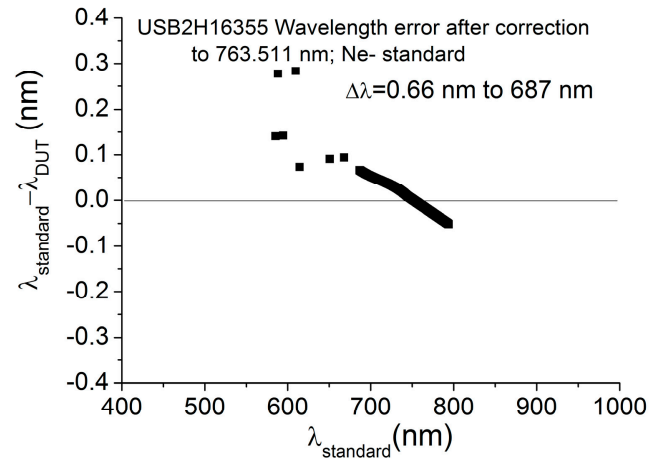

(d)

Figure 11. The wavelength corrections for (a) Ar and (b) Ne lamps' emission lines measured with spectrometer USB2H1635 ( $\lambda_{\text {DUT }}$; DUT, device under test) after optimization at $763.511 \mathrm{~nm}$ and the corresponding errors $(\mathbf{c}, \mathbf{d})$ related to the standard values $\left(\lambda_{\text {standard }}\right)$. The red lines represent the standard emission lines for Ar and Ne lamps.

Table 2. The wavelength differences between Piccolo-corrected wavelength values and the standard Ne and Ar emission lines for spectrometers QEP00114, QEP00981 and USB2H1635.

\begin{tabular}{|c|c|c|c|c|c|c|c|c|}
\hline \multirow[t]{2}{*}{ DUT } & \multicolumn{4}{|c|}{ Ar } & \multicolumn{4}{|c|}{$\mathrm{Ne}$} \\
\hline & $\lambda_{\mathrm{o}}$ & $\lambda_{\text {ref }}$ & $\lambda_{\text {error. }}$ & FWHM & $\lambda_{\mathrm{o}}$ & $\lambda_{\text {ref }}$ & $\lambda_{\text {error. }}$ & FWHM \\
\hline & $\mathrm{nm}$ & $\mathrm{nm}$ & $\mathrm{nm}$ & $\mathrm{nm}$ & $\mathrm{nm}$ & $\mathrm{nm}$ & $\mathrm{nm}$ & $\mathrm{nm}$ \\
\hline \multirow[t]{8}{*}{ QE00114 } & $696 . .431$ & 696.543 & 0.112 & 0.293 & 653.080 & 653.290 & 0.210 & 0.388 \\
\hline & 706.639 & 706.722 & 0.082 & 0.366 & 667.637 & 667.830 & 0.193 & 0.301 \\
\hline & 727.252 & 727.294 & 0.042 & 0.439 & 671.522 & 671.700 & 0.178 & 0.290 \\
\hline & 738.368 & 738.398 & 0.030 & 0.435 & 692.825 & 692.950 & 0.125 & 0.504 \\
\hline & 750.374 & 750.387 & 0.013 & 0.429 & 724.479 & 724.520 & 0.041 & 0.379 \\
\hline & 763.511 & 763.511 & 0.000 & 0.429 & - & - & - & - \\
\hline & 772.4367 & 772.376 & -0.061 & 0.422 & - & - & - & - \\
\hline & 794.851 & 794.818 & -0.033 & 0.331 & - & - & - & - \\
\hline \multirow[t]{12}{*}{ QE00981 } & 696.326 & 696.543 & 0.2170 & 1.941 & 587.390 & 588.190 & 0.800 & 1.446 \\
\hline & 706.553 & 706.722 & 0.1690 & 1.943 & 593.794 & 594.480 & 0.686 & 1.720 \\
\hline & 727.221 & 727.294 & 0.0729 & 1.999 & 613.941 & 614.310 & 0.369 & 1.514 \\
\hline & 738.356 & 738.398 & 0.0420 & 2.017 & 650.724 & 650.650 & -0.074 & 1.732 \\
\hline & 750.873 & 750.387 & -0.4860 & 2.554 & 668.030 & 667.830 & -0.200 & 1.791 \\
\hline & 763.511 & 763.511 & 0.0000 & 1.998 & 693.332 & 692.950 & -0.382 & 1.902 \\
\hline & 772.429 & 772.376 & -0.0539 & 1.964 & 725.050 & 724.520 & -0.530 & 1.980 \\
\hline & 794.857 & 794.818 & -0.0388 & 1.966 & - & - & - & - \\
\hline & 811.36 & 811.531 & 0.171 & 2.461 & - & - & - & - \\
\hline & 826.467 & 826.452 & -0.015 & 2.071 & - & - & - & - \\
\hline & 841.938 & 842.465 & 0.5272 & 3.179 & - & - & - & - \\
\hline & 912.031 & 912.297 & 0.2656 & 2.307 & - & - & - & - \\
\hline \multirow[t]{11}{*}{ USB2H1635 } & 5696.479 & 696.543 & 0.063 & 1.651 & 585.120 & 585.260 & 0.14 & 1.820 \\
\hline & 706.678 & 706.722 & 0.044 & 1.367 & 587.913 & 588.190 & 0.277 & 1.907 \\
\hline & 727.266 & 727.294 & 0.028 & 1.693 & 594.338 & 594.480 & 0.142 & 1.720 \\
\hline & 738.369 & 738.398 & 0.028 & 1.684 & 609.337 & 609.620 & 0.283 & 1.700 \\
\hline & 750.920 & 750.387 & -0.533 & 2.378 & 614.236 & 614.310 & 0.074 & 1.936 \\
\hline & 763.511 & 763.511 & 0 & 1.705 & 650.559 & 650.650 & 0.091 & 1.519 \\
\hline & 772.416 & 772.376 & -0.04 & 1.670 & 667.736 & 667.830 & 0.094 & 1.665 \\
\hline & 811.437 & 811.531 & 0.094 & 2.135 & 692.890 & 692.950 & 0.06 & 1.697 \\
\hline & 826.533 & 826.452 & -0.081 & 1.833 & 724.487 & 724.520 & 0.033 & 1.672 \\
\hline & 842.127 & 842.465 & 0.338 & 2.900 & 743.881 & 743.890 & 0.009 & 1.576 \\
\hline & 912.117 & 912.297 & 0.181 & 1.458 & - & - & - & - \\
\hline
\end{tabular}

$\lambda_{\mathrm{o}}$, wavelength corrected after optimization at $763.511 \mathrm{~nm} ; \lambda_{\text {ref }}$, standard wavelength; $\lambda_{\text {error., }}$ wavelength difference between the standard emission line $\lambda_{\text {ref }}$ and $\lambda_{\mathrm{o}}$. 


\subsection{Field Spectrometers Spectral Characterization with Monochromator Light Source}

As an alternative to emission lamps to calibrate the spectrometers at the telluric oxygen absorption lines, a second wavelength standard, an OL750D double monochromator system from Gooch \& Housego, was selected. Since for high spectral resolution wavelength calibration, it is important that the wavelength standard have low uncertainty $<0.1 \mathrm{~nm}$, this uncertainty can be only assessed with high accuracy if the monochromator's FWHM and sampling steps are known. Therefore, the OL750D double monochromator had to be characterised before spectrometer wavelength and FWHM characterisation could be carried out.

\subsubsection{Monochromator Light Source Characterization with Reference Emission Lamp}

The double monochromator system (Gooch \& Housego OL750D) spectral capabilities were evaluated using the technique presented in Section 3.1. Instead of spectrometers measuring the Ar and Ne gas lamps, the monochromator system was used with different configurations. These configurations are presented in Table 3. A Gaussian fit was again applied to obtain the centre wavelength and the spectral resolution for each wavelength scanning interval selected. Values between $0.07 \mathrm{~nm}$ and $3.5 \mathrm{~nm}$ FWHM were obtained (Figure 12), and the highest spectral resolution corresponded to $0.05 \mathrm{~mm}$ slits width. However, with this configuration $(0.05 \mathrm{~mm}$ slits width), a spectral response asymmetry was observed (Figure 12g,h).

Table 3. Wavelength correction values for different slits configurations of double monochromator OL750D.

\begin{tabular}{ccccccccc}
\hline & \multicolumn{4}{c}{ Ar } & \multicolumn{4}{c}{ Ne } \\
\hline OL750D Slits & $\lambda_{\text {meas }}$ & FWHM & $\lambda_{\text {ref }}$ & $\lambda_{\text {corr. }}$ & $\lambda_{\text {meas }}$ & FWHM & $\lambda_{\text {ref }}$ & $\lambda_{\text {corr. }}$ \\
\hline $\mathbf{~ m m ~}$ & $\mathbf{n m}$ & $\mathbf{n m}$ & $\mathbf{n m}$ & $\mathbf{n m}$ & $\mathbf{n m}$ & $\mathbf{n m}$ & $\mathbf{n m}$ & $\mathbf{n m}$ \\
\hline $0.05,0.5$ and 0.05 & 763.855 & 0.077 & 763.511 & -0.344 & 693.223 & 0.045 & 692.95 & -0.273 \\
$1.25,5$ and 1.25 & 763.552 & 0.969 & 763.511 & -0.041 & 693.004 & 1.066 & 692.95 & -0.054 \\
$2.5,5$ and 2.5 & 763.561 & 1.813 & 763.511 & -0.05 & 693.022 & 2.014 & 692.95 & -0.072 \\
5,5 and 5 & 763.534 & 3.161 & 763.511 & -0.023 & 692.994 & 3.519 & 692.95 & -0.044 \\
\hline
\end{tabular}

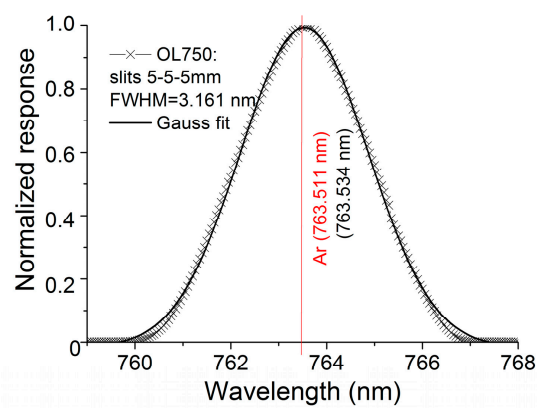

(a)

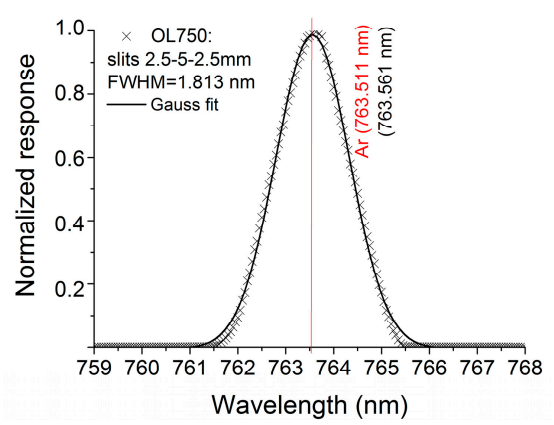

(c)

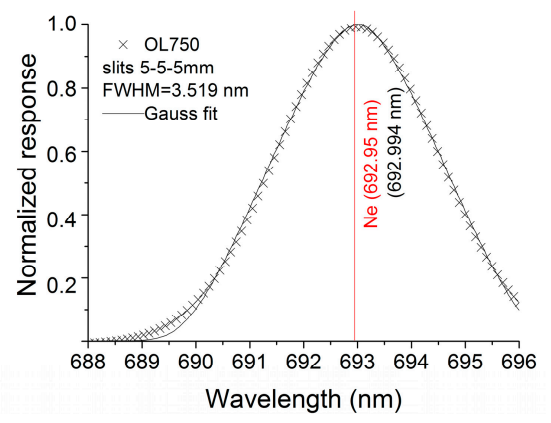

(b)

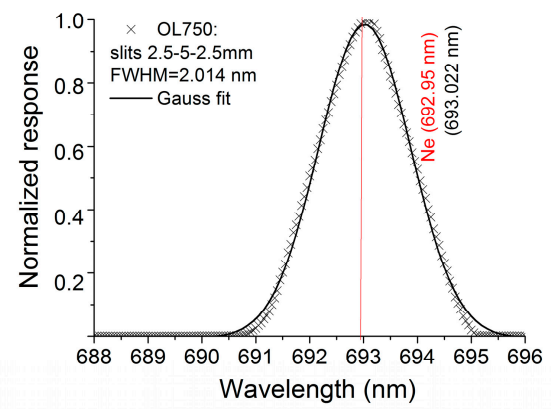

(d)

Figure 12. Cont. 


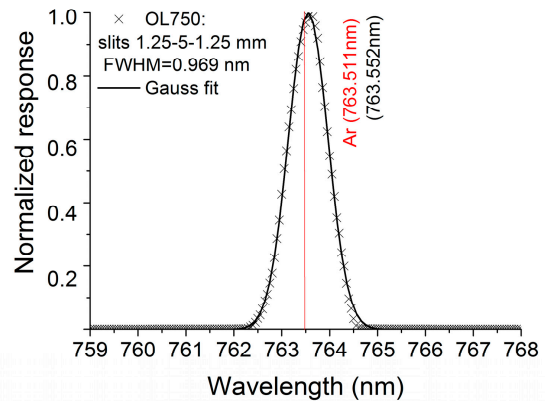

(e)

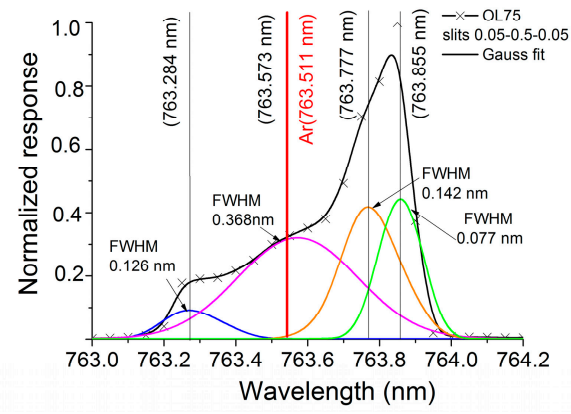

(g)

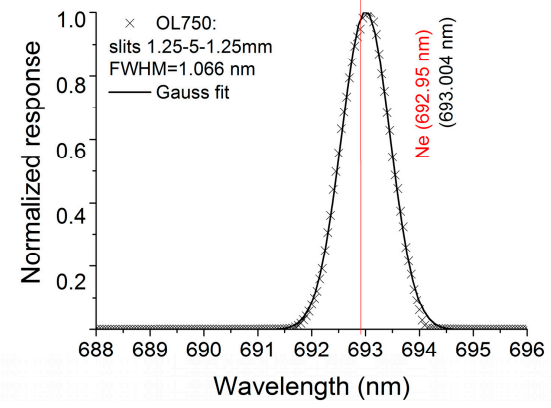

(f)

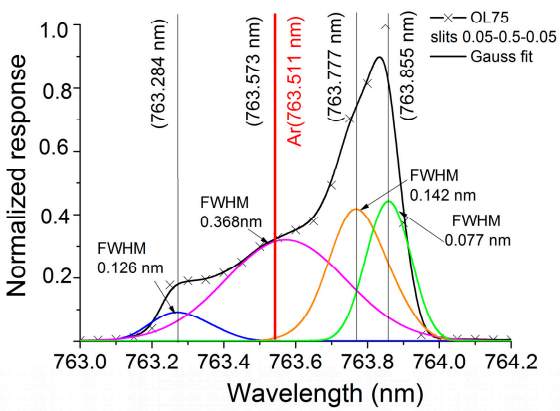

(h)

Figure 12. Ne (left) and Ar (right) lamps' emission line measured with the double monochromator system OL 750D from Gooch \& Housego and the corresponding standard absorption lines (red lines) [34] for slits' combination at the monochromator entrance, middle and exit: (a,b) $5 \mathrm{~mm}$, $5 \mathrm{~mm}$ and $5 \mathrm{~mm}$; (c,d) $2.5 \mathrm{~mm}, 5 \mathrm{~mm}$ and $2.5 \mathrm{~mm}$; (e,f) $1.25 \mathrm{~mm}, 5 \mathrm{~mm}$ and $1.25 \mathrm{~mm}$; (h,f) $0.05 \mathrm{~mm}$, $0.5 \mathrm{~mm}$ and $0.05 \mathrm{~mm}$. The coloured lines represent the Gaussian fit applied to the multiple peaks under the envelope

The wavelength error was determined from the wavelength difference between $\mathrm{Ne}$ and $\mathrm{Ar}$ standard emission lines and the measured values with OL 750. The error values were between $0.02 \mathrm{~nm}$ and $0.3 \mathrm{~nm}$ (Table 3) with the smaller slits displaying the larger errors. These values were applied to correct the monochromator measured wavelength values. Accordingly, correction factors of $-0.344 \mathrm{~nm},-0.041 \mathrm{~nm}$ and $-0.05 \mathrm{~nm}$ were applied to correct the monochromator system wavelength to the 763.511-nm Ar emission line when 0.05- $\mathrm{mm}, 1.25-\mathrm{mm}$ and 2.5-mm slit width configurations were used, respectively. The monochromator calibration is also limited to reference lamp emission lines; therefore, as with the field spectrometers, it too cannot be calibrated at the fluorescence emission lines.

\subsubsection{Field Spectrometers Spectral Characterization with Monochromator Light Source}

The monochromator has the advantage of spectral resolution tuneability (Figure 12); therefore, the OL750D with a slit width of $1.25 \mathrm{~nm}$ can be used for reflectance spectrometers' spectral calibration, and the $0.05 \mathrm{~nm}$ slit width can be used to calibrate the fluorescence spectrometers.

Here, the results obtained for the QEP00114 spectrometer (DUT) spectral calibration to multiple wavelengths around $687 \mathrm{~nm}$ (corresponding to O2-A absorption line) and $760 \mathrm{~nm}$ (corresponding to $\mathrm{O} 2-\mathrm{B}$ line) using as the wavelength standard the OL750D with the $0.05 \mathrm{~mm}$ entrance and exit slits and $0.5 \mathrm{~mm}$ in the middle (Figure 13) are presented. The correction values vary along the scanning interval within less than $0.02 \mathrm{~nm}$. The measured wavelength values for DUT represent the mean values of 10 acquired spectra, with a maximum spectral response Type A uncertainty of $0.12 \%$ along the measured spectral range. A wavelength correction of $1.11 \mathrm{~nm}$ was applied for $687 \mathrm{~nm}$ and of $1.037 \mathrm{~nm}$ for $760 \mathrm{~nm}$. These values are similar to those found when line lamps were used as the wavelength standard (the wavelength correction was $0.981 \mathrm{~nm}+0.14 \mathrm{~nm}=1.121 \mathrm{~nm}$ when using Ar and Ne lamps). 


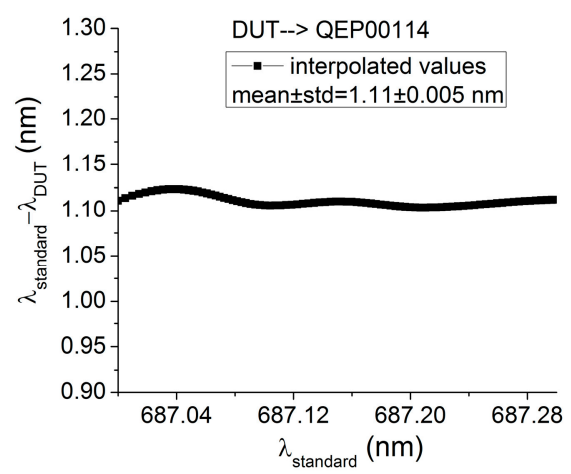

(a)

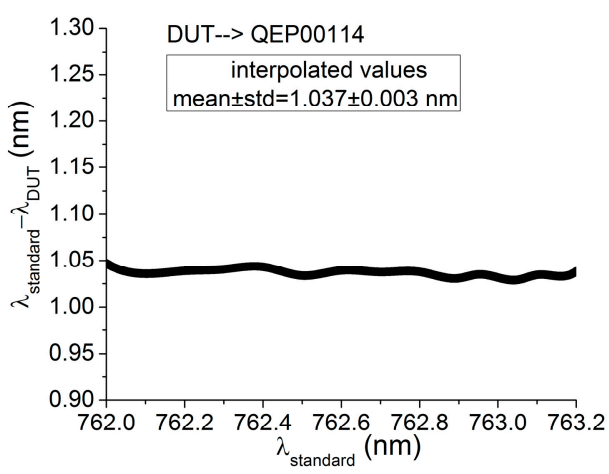

(b)

Figure 13. The fluorescence spectrometer wavelength correction within the spectral ranges (a) $680 \mathrm{~nm}$ to 687.3 and (b) $762 \mathrm{~nm}$ to $763.2 \mathrm{~nm}$, using the high-resolution configuration of OL750D.

\subsubsection{Fluorescence Spectrometer QEP00114 Spectral Resolution}

Using both wavelength emission line standards, it was possible to characterize the spectral resolution of spectrometers again using a Gaussian fit. The spectrometers FWHM corresponding to gas lamp emission were determined using the pen-line lamps, and the spectrometers' spectral capabilities at the wavelength corresponding to the oxygen telluric lines were determined using the double monochromator system. An example of the spectral capabilities of QEP00114, the fluorescence spectrometer, determined from line lamps and monochromator system source, is presented in Figure 14.

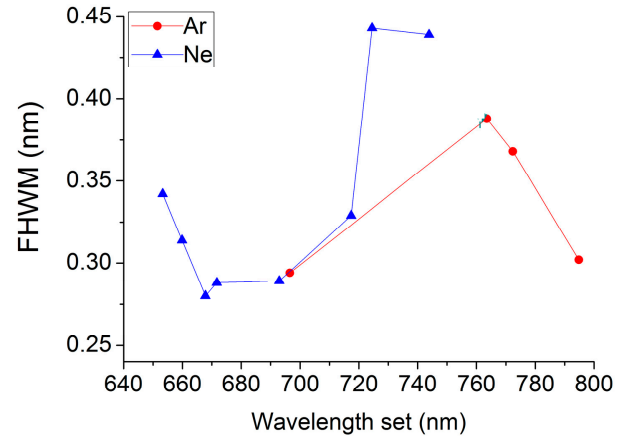

(a)

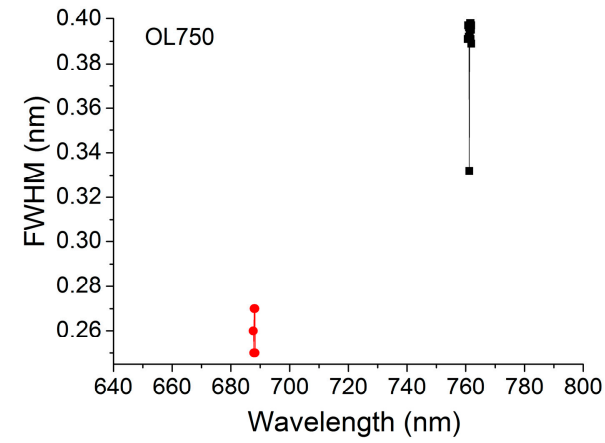

(b)

Figure 14. The FWHM values as determined from the Gaussian fit applied to the QEP00114 signal corresponding to the (a) Ar/Ne and (b) OL750D emission.

\section{Discussion}

Two approaches have been used to assess the wavelength calibration and spectral response of three Ocean Optics spectrometers commonly used in fluorescence and reflectance studies. In the first case, emission reference lamps were used to optimize the wavelength calibration with the Ar emission lamp feature at $763.511 \mathrm{~nm}$, the closest emission line to the O2-A absorption band. When using this correction, an error of $0.14 \mathrm{~nm}$ was noted for the QEP00981 spectrometer at the O2-B line centred at $687 \mathrm{~nm}$, after data B-spline interpolation. For USB2H1635, the errors values varied within $0.4 \mathrm{~nm}$ over the entire selected spectral range and within $0.66 \mathrm{~nm}$ for the QEP00981 spectrometer. 
To calibrate the wavelength scale of their spectrometers, Ocean Optics recommend using a third order polynomial fit to establish the relationship between a number of pixels and the emission lines of a Hg-Ar lamp [37]. This polynomial is then used to predict and assign a wavelength value to all pixels. From this process, the difference between the predicted wavelengths and the reference emission source wavelengths can be determined. The errors at each of the emission line wavelengths can then be tabulated and, as the example in [37] shows, can be seen to be distributed across the spectral range of the spectrometer where emission lines are present. However, for spectrometers for fluorescence investigation having a spectral range in the spectral range from $640 \mathrm{~nm}-800 \mathrm{~nm}$, few $\mathrm{Hg}$-Ar or Ar emission lines are present. The polynomial derived will therefore be less accurate as there are fewer points to determine the fit. The polynomial also does not optimise the calibration at or near the wavelengths of interest for these fluorescence studies, but distributes the calibration coefficients across the entire spectral range of the spectrometer. By selecting other emission lamps with other gasses (i.e., Ne), additional emission lines can be used for the fit, and these might be closer to the wavelengths of interest for fluorescence studies. If multiple emission sources are employed, they could be measured independently, then the data compiled and analysed simultaneously. This would enable emission lines across the 579-nm-696-nm region, for example, to be included in the polynomial fit as emission lines are present in this region in Ne gas. However, it is worth noting that the change in sampling interval and in FWHM across the spectral ranges of each Ocean Optics spectrometer is a characteristic of the Czerny-Turner optical bench design as the light impinging on the diffraction grating is not perpendicular to the grating across its full width. As such, they cannot be avoided, but should be characterized and reported.

The use of a monochromator system as a wavelength standard instead of line lamps was also investigated in this work. First, the monochromator was characterized in spectral response using the same technique like in the case of the field spectrometer, being employed as standard wavelengths the Ar and Ne line lamps. Combining different slit widths and different light sources, the spectral resolution of the monochromator was tuned between $<0.1 \mathrm{~nm}$ and $3.5 \mathrm{~nm}$. It was observed that for lower FWHM values, the error increased, when compared with the standard values. The highest error was in the case of $0.05-\mathrm{mm}$ slits, when a peak asymmetry was also noticed. The peak asymmetry can induce an uncertainty on the centre wavelength determination. We presume that this asymmetry is the result of either the misalignment of the LDLS light source to the monochromator entrance or due to the diffractions associated with slit width. A more detailed monochromator system characterization, for all components (slits width between 1.25 and $0.05 \mathrm{~nm}$, light source, gratings, detector) will need to be considered in the future to understand the source of this error and to reduce it.

After OL750D wavelength calibration, the system was used as the wavelength transfer standard to calibrate the field spectrometers at $687 \mathrm{~nm}$ and $760 \mathrm{~nm}$. The wavelength correction was similar for the QEP00114 fluorescence spectrometer when two different calibration sources were used: $+1.121 \mathrm{~nm}$ when the pen-ray lamp was considered as the standard and $+1.11 \mathrm{~nm}$ in the case of the monochromator system (with a maximum standard deviation of $\pm 0.005 \mathrm{~nm}$ ). The advantage of using the monochromator source is that the wavelength calibration can be performed at specific lines corresponding to chlorophyll fluorescence emission, and not at fixed lines as in the case of the reference lamp used. Furthermore, the spectral features of the spectrometer related to the FWHM can be determined at any desired wavelength when using a double monochromator source with high accuracy if the system is fully characterized as has been demonstrated in this paper. 
Considering all of the above-mentioned issues, we may say that the following options are available to correct the spectrometers spectral data to be used for fluorescence measurements:

- $\quad$ to calculate the wavelength errors considering the difference between each standard line (NIST database) and the measured spectral line; in this way, a wavelength correction of $\pm 3.2 \mathrm{~nm}$ will be applied to all data, with an accuracy of $\pm 0.3 \mathrm{~nm}$ (see Figures 3 and 4);

- $\quad$ to optimise the wavelength correction close to O2-A and / or O2-B features, obtaining minimum error at specific wavelengths, but higher error outside this range. This method still relies on a polynomial fit. This method can be a better option for high resolution spectrometers used for SIF studies. If the correction is optimised to only one wavelength close to the O2-A absorption line, an error of $\pm 0.125 \mathrm{~nm}$ (Table 2) is obtained for the O2-B line. Therefore, it is highly recommended to have wavelength correction at both telluric bands using an additional line lamp (e.g., $\mathrm{Ne}$ ) to minimize the error. Furthermore, the double monochromator could be used at any wavelength, but an uncertainty due to limited standard calibration has to be considered.

A better accuracy for a larger spectral range can be reached by using a tuneable laser as a spectral standard.

As concerns the uncertainties of the sources in the case of field spectrometers' spectral calibration, the temperature variation effect has to be addressed. Two aspects have to be considered: either the sensor is thermally controlled or the entire system is cooled. If only the detector is temperature controlled, then a wavelength shifts can appear due to the thermal expansion of optical and mechanical parts. For field spectrometers, it is strongly recommended to wait for a thermal stabilization of the instruments according to the manufacturer's recommendations [18]. A compact design of the spectrometer's optical setup, a lack of moving parts and the appropriate selection of materials with low expansion coefficients $\left( \pm 0.01\right.$ to $\pm 0.08 \mathrm{~nm} /{ }^{\circ} \mathrm{C}$ [38] ) assure a good reproducibility of wavelength measurements and provide a low wavelength shift (e.g., \pm 0.17 pixel $/{ }^{\circ} \mathrm{C}$ for the high resolution AvaSpec-ULS3648, having a pitch of $8 \mu \mathrm{m}$ and operating in the 600-700-nm spectral band [39]).

In relation to the optical signal coupling to the spectrometers, additional precautions have to be taken in order to reduce the wavelength calibration errors: (i) the numerical aperture (NA) of the coupling optical fibre has to be larger than the NA of the instrument; (ii) for spectrometers having entrance slit widths less than $70 \mu \mathrm{m}$, it is recommended to select an optical fibre core three-times larger than the slit width. A mismatch between the connecting optical fibre and the spectrometer input optics can lead either to a wavelength shift or to the decrease in spectral data reproducibility [38]. Furthermore, a misalignment of the light source to the monochromator input port can distort the shape of the spectral line symmetry.

\section{Conclusions}

This paper presents the results obtained from spectral calibration of three field spectrometers configured for fluorescence and reflectance measurements. Two different approaches were described for this purpose. In the first case, two different standard pen-ray line lamps, having well-known spectral features, were used as wavelength standards, and for the second case, a monochromator-based system was developed to calibrate the spectrometers.

One may conclude from the results is that if the first method is selected, either the wavelength correction is performed for multiple emission lines corresponding to the standard line-lamps and an accuracy of $\pm 0.3 \mathrm{~nm}$ is obtained for the entire spectral range (Figures 3 and 4), or the spectral corrections are optimized at emission lines close to telluric oxygen Bands $\mathrm{A}$ and $\mathrm{B}$, using a combination of line lamps (e.g., Ar/Hg-Ar and Ne). Still, these gas lamps have not enough spectral features in the desired range. This issue could be partially solved using a monochromator system instead that can be tuned to characterize and calibrate the spectrometers to specific wavelengths.

The characterization of a double monochromator was presented here for different slit width dimensions to obtain different spectral resolutions. However, this would be time consuming, 
and further work is required to optimise the sampling interval and interpolation method if it were not to be done pixel by pixel. Thus, further work is required for a better characterization of the OL750 monochromator system, particularly at the highest resolutions (lowest FWHM) and identifying asymmetries.

Therefore, we may conclude that employing either the line-lamp or the double monochromator standards for field spectrometers' spectral calibration, the wavelength correction is limited by the availability of appropriate emission lines of the gas lamps (monochromator being calibrated using line-lamps also). There is a gap in the spectral calibration over the $\mathrm{O} 2$ telluric bands, a gap that could be covered using a tuneable laser source. The use of a tuneable laser for this purpose is the subject of our future projects.

Finally, it was noted during this work that the sampling intervals and FWHM of these spectrometers changed across their spectral range. These aspects of spectrometer performances have implications on the choice of instrument and the performance specifications that are required to enable reliable and replicable measurements of fluorescence signals with quantifiable accuracies and precision.

The steps suggested in this paper could be replicated at various facilities and laboratories for different types of spectrometer systems, such as the FLEX fluorescence imager, at a sampling interval and band widths that make the detection of the solar flux in the telluric oxygen $\mathrm{A}$ and $\mathrm{B}$ bands possible.

Acknowledgments: The authors would like to acknowledge the support of Chris MacLellan from the NERC/NCEO, Field Spectroscopy Facility, GeoSciences, University of Edinburgh, for assistance in making the measurement, understanding the operation and increasing the resolution of the Gooch \& Housego OL750. The authors also wish to acknowledge the support of the COST Association (with EU H2020 funding support) and COST Action ES1309 (OPTIMISE) for supporting Mihai's short-term scientific mission to the Field Spectroscopy Facility and for forming the network that has enabled the collaboration of the authors of this paper. The Romanian authors acknowledge the financial support of the Romanian Space Agency under Contract 131/2017.

Author Contributions: L.M. elaborated the manuscript and performed the experiments and data processing. A.M.A. and D.S. initiated the experiment, designed the setup and test procedures and participated in the manuscript preparation. A.H. helped in the interpretation of the results and reviewed the manuscript. I.R. performed the Piccolo acquisition routines.

Conflicts of Interest: The authors declare no conflict of interest.

\section{References}

1. Baker, N.R. Chlorophyll fluorescence: A probe of photosynthesis in vivo. Annu. Rev. Plant Biol. 2008, 59, 89-113. [CrossRef] [PubMed]

2. Papageorgiou, G.C. (Ed.) Chlorophyll a Fluorescence: A Signature of Photosynthesis; Advances in Photosynthesis and Respiration; Springer: Dordrecht, The Netherlands, 2004; Volume 19, pp. 1-42. ISBN 978-1-4020-3217-2.

3. European Space Agency (ESA). Report for Mission Selection: FLEX, ESA SP-1330/2; European Space Agency: Noordwijk, The Netherlands, 2015; ISBN 978-92-9221-428-9.

4. Maxwell, K.; Johnson, G.N. Chlorophyll fluorescence-A practical guide. J. Exp. Bot. 2000, 51, $659-668$. [CrossRef] [PubMed]

5. Jauffraud, E.; Bassaler, P.; Coppo, P.; Taiti, A.; Battistelli, E.; Rossi, M. FLEX \& Sentinel 3: A tandem to monitor vegetation. In Proceedings of the 2016 European Space Agency Living Planet Symposium, Prague, Czech Republic, 9-13 May 2016.

6. ESA Website. Available online: https://earth.esa.int/web/guest/missions/esa-future-missions/flex/news/ - / article/sentinels-and-flex-join-forces (accessed on 10 November 2017).

7. Website of Workshop on Land Product Validation and Evolution (LPVE 2018). Available online: https:/ / nikal.eventsair.com/QuickEventWebsitePortal/lpve-conference-2018/esaconference/ExtraContent/ ContentPage?page=1 (accessed on 10 November 2017).

8. Schaepman, M. Calibration of a Field Spectroradiometer. Ph.D. Thesis, University of Zurich, Zürich, Switzerland, 1998.

9. Kohler, D.D.R.; Bissett, W.P.; Steward, R.G.; Davis, C.O. New approach for the radiometric calibration of spectral imaging systems. Opt. Express 2004, 12, 2463-2477. [CrossRef] [PubMed] 
10. Klaasen, K.P.; A’Hearn, M.F.; Baca, M.; Delamere, A.; Desnoyer, M.; Farnham, T.; Groussin, O.; Hampton, D.; Ipatov, S.; Lisse, J.; et al. Invited article: Deep impact instrument calibration. Rev. Sci. Instrum. 2008, 79, 091301. [CrossRef] [PubMed]

11. Schaepman, M.E.; Dangel, S. Solid laboratory calibration of a nonimaging spectroradiometer. Appl. Opt. 2000, 39, 3754-3764. [CrossRef] [PubMed]

12. Meroni, M.; Rossini, M.; Guanter, L.; Alonso, L.; Rascher, U.; Colombo, R.; Moreno, J. Remote sensing of solar induced chlorophyll fluorescence: Review of methods and applications. Remote Sens. Environ. 2009, 113, 2037-2051. [CrossRef]

13. Joiner, J.; Guanter, L.; Lindstrot, R.; Voigt, M.; Vasilkov, A.P.; Middleton, E.M. Global monitoring of terrestrial chlorophyll fluorescence from moderate spectral resolution near-infrared satellite measurements: Methodology, simulations, and application to GOME-2. Atmos. Meas. Tech. 2013, 6, 3883-3930. [CrossRef]

14. Guanter, L.; Rossini, M.; Colombo, R.; Meroni, M.; Frankenberg, C.; Lee, J.-E.; Joiner, J. Using field spectroscopy to assess the potential of statistical approaches for the retrieval of sun-induced chlorophyll fluorescence from ground and space. Remote Sens. Environ. 2013, 133, 52-61. [CrossRef]

15. Meroni, M.; Busetto, L.; Colombo, R.; Guanter, L.; Moreno, J.; Verhoef, W. Performance of spectral fitting methods for vegetation fluorescence quantification. Remote Sens. Environ. 2010, 114, 363-374. [CrossRef]

16. Damm, A.; Erler, A.; Hillen, W.; Meroni, M.; Schaepman, M.E.; Verhoef, W.; Rascher, U. Modeling the impact of spectral sensor configurations on the FLD retrieval accuracy of sun-induced chlorophyll fluorescence. Remote Sens. Environ. 2011, 115, 1882-1892. [CrossRef]

17. Ding, W.; Zhao, F.; Yang, L. Analyzing the impact of sensor characteristics on retrieval methods of solar-induced fluorescence. In Proceedings of the SPIE International Conference on Optical and Photonics Engineering (icOPEN 2016), Chengdu, China, 26-30 September 2016; Volume 10250.

18. Hueni, A.; Damm, A.; Kneubühler, M.; Schläpfer, D.; Schaepman, M.E. Field and airborne spectroscopy cross validation-Some considerations. IEEE J. Sel. Top. Appl. Earth Obs. Remote Sens. 2017, 10, 1117-1135. [CrossRef]

19. Guanter, L.; Alonso, L.; Gomez-Chova, L.; Meroni, M.; Preusker, R.; Fischer, J.; Moreno, J. Developments for vegetation fluorescence retrieval from spaceborne high-resolution spectrometry in the O2-A and O2-B absorption bands. J. Geophys. Res. Atmos. 2010, 115. [CrossRef]

20. Rossini, M.; Meroni, M.; Migliavacca, M.; Manca, G.; Cogliati, S.; Busetto, L.; Picchi, V.; Cescatti, A.; Seufert, G.; Colombo, R. High resolution field spectroscopy measurements for estimating gross ecosystem production in a rice field. Agric. For. Meteorol. 2010, 150, 1283-1296. [CrossRef]

21. Meroni, M.; Barducci, A.; Cogliati, S.; Castagnoli, F.; Rossini, M.; Busetto, L.; Migliavacca, M.; Cremonese, E.; Galvagno, M.; Colombo, R.; et al. The hyperspectral irradiometer, a new instrument for long-term and unattended field spectroscopy measurements. Rev. Sci. Instrum. 2011, 82, 043106. [CrossRef] [PubMed]

22. Cogliati, S.; Rossini, M.; Julitta, T.; Meroni, M.; Schickling, A.; Burkart, A.; Pinto, F.; Rascher, U.; Colombo, R. Continuous and long-term measurements of reflectance and sun-induced chlorophyll fluorescence by using novel automated field spectroscopy systems. Remote Sens. Environ. 2015, 164, 270-281. [CrossRef]

23. Buschmann, C.; Nagel, E.; Szabó, K.; Kocsányi, L. Spectrometer for fast measurements of in vivo reflection, absorption and fluorescence in the visible and near infrared. Remote Sens. Environ. 1994, 48, 18-24. [CrossRef]

24. Julitta, T.; Rossini, M.; Burkart, A.; Cogliati, S.; Davies, N.; Hom, M.; Mac Arthur, A.; Middleton, E.M.; Rascher, U.; Schickling, A.; et al. Comparison of sun-induced chlorophyll fluorescence estimates obtained from four portable field spectroradiometers. Remote Sens. 2016, 8, 122. [CrossRef]

25. Basics of Spectral Measurement. JETI Technische Instrumente GmbH. Available online: www.jeti.com/cms/ images/jeti_com/down/basics/basics.pdf (accessed on 20 January 2018).

26. Ocean Optics Website. Available online: https://oceanoptics.com/wp-content/uploads/SpectrometerWavelength-Calibration-Instructions.pdf (accessed on 20 January 2018).

27. Yoon, H.W.; Kacker, R.N. Handbook (NIST HB)-157; NIST Publications: Gaithersburg, MD, USA, 2015.

28. Alonso, L.; Gómez-Chova, L.; Vila-Francés, J.; Amorós-López, J.; Guanter, L.; Calpe, J.; Moreno, J. Improved Fraunhofer Line Discrimination method for vegetation fluorescence quantification. IEEE Geosci. Remote Sens. Lett. 2008, 5, 620-624. [CrossRef]

29. Choi, K.Y.; Milton, E.J. Estimating the spectral response function of the CASI-2. In Proceedings of the Annual Conference of the Remote Sensing and Photogrammetry Society, Falmouth, UK, 15-17 September 2008. 
30. Liu, L.; Liu, X.; Hu, J. Effects of spectral resolution and SNR on the vegetation solar-induced fluorescence retrieval using FLD-based methods at canopy level. Eur. J. Remote Sens. 2015, 48, 743-762. [CrossRef]

31. Centre of Nanotechnology and Materials for Nanoelectronics Website. Available online: http:/ / nanotech. fzu.cz/26/data/PenRay.pdf (accessed on 31 January 2018).

32. Pen-Ray Line Sources. Available online: http://pas.ce.wsu.edu/CE415/PenRay_lamp_spectra.pdf (accessed on 31 January 2018).

33. Mac Arthur, A.; Robinson, I.; Rossini, M.; Davis, N.; MacDonald, K. A dual-field-of-view spectrometer system for reflectance and fluorescence measurements (Piccolo Doppio) and correction of etaloning. In Proceedings of the 5th International Workshop on Remote Sensing of Vegetation Fluorescence, Paris, France, 22-24 April 2014.

34. Kramida, A.; Ralchenko, Y.; Reader, J.; NIST ASD Team. NIST Atomic Spectra Database (Version 5.5.2); National Institute of Standards and Technology: Gaithersburg, MD, USA, 2018. Available online: https:/ / physics.nist.gov/asd (accessed on 29 January 2018).

35. Reader, J.; Sansonetti, C.J.; Bridges, J.M. Irradiances of spectral lines in mercury pencil lamps. Appl. Opt. 1996, 35, 78-83. [CrossRef] [PubMed]

36. Wang, Z.; Wang, K.; An, S. Cubic B-Spline Interpolation and Realization. In Communications in Computer and Information Science, Proceedings of the Information Computing and Applications (ICICA 2011), Qinhuangdao, China, 28-31 October 2011; Liu, C., Chang, J., Yang, A., Eds.; Springer: Berlin/Heidelberg, Germany, 2011; Volume 43.

37. Ocean Optics. Calibrating the Wavelength of the Spectrometer n.d. Available online: https:/ /oceanoptics.com/ wp-content/uploads/Spectrometer-Wavelength-Calibration-Instructions.pdf (accessed on 31 January 2018).

38. Mini-Spectrometers. Hamamatsu. Available online: https://www.hamamatsu.com/resources/pdf/ssd/ mini-spectrometer_kacc9003e.pdf (accessed on 31 January 2018).

39. Avantes Catalog, X. Available online: https://www.avantes.com/support/downloads/catalog/356-avantescatalog-x/file (accessed on 31 January 2018).

(C) 2018 by the authors. Licensee MDPI, Basel, Switzerland. This article is an open access article distributed under the terms and conditions of the Creative Commons Attribution (CC BY) license (http:/ / creativecommons.org/licenses/by/4.0/). 